\title{
Assessment of Wet Age-Related Macular degeneration by Optical Coherence Tomography Angiography
}

Sayed A. Sayed, Hossam Eldin A. Ziada, Mahmoud S. Mohammad*

Department of Ophthalmology, Faculty of Medicine, Al-Azhar University, Cairo, Egypt

*Corresponding author: Mahmoud S. Mohammad, E-mail: mygoodhayat@gmail.com, Telephone No.: +2001005750332

\begin{abstract}
Background: to date, fundus fluorescein angiography (FFA), remains the gold standard for the diagnosis of choroidal neovascular membrane (CNV). Optical coherence tomography angiography (OCTA) is a new imaging modality that allows a clear, depth-resolved visualization of the retinal vascular structures.

Aim of the Work: to evaluate the role of OCTA in diagnosis of CNV in patients with age-related macular degeneration (AMD).

Patients and Methods: this study enrolled 32 patients (40 eyes), including 20 patients (20 eyes) with wet AMD and 12 patients (20 eyes) with dry AMD. All patients underwent swept-source optical coherence tomography (SS-OCT), swept-source OCTA, and fundus fluorescein angiography (FFA). OCTA was used to evaluate neovascular networks in terms of their type, location and extent of visualization. Sensitivity and specificity of the method were assessed separately in a group of $20 \mathrm{CNV}$ eyes and 20 dry AMD based on FFA diagnosis as the gold standard.
\end{abstract}

Results: sensitivity and specificity of en face OCTA were $95 \%$ and $100 \%$ respectively. Both Sensitivity and specificity of structural OCT were $100 \%$.

Conclusion: OCTA enables diagnosis of both classic and occult choroidal neovascularization in patients with AMD. The method has high sensitivity and specificity.

Keywords: optical coherence tomography angiography, age-related macular degeneration, choroidal neovascularization.

\section{INTRODUCTION}

Age-related macular degeneration (AMD) is the term applied to ageing changes without any other obvious precipitating cause that occur in macula in people aged 50 years and above (1). Aging is the strongest risk factor for AMD, but, of course, it is not modifiable. Age-related changes in Bruch's membrane and age-related formation of the components of drusen play the strongest role in AMD ${ }^{(2)}$. AMD ranks third among the causes of blindness, following cataract and glaucoma. However, it is the primary cause of blindness in industrialized nations ${ }^{(3)}$. AMD has been classified depending on whether there is a presence of abnormal neovascularization into wet (exudative or neovascular) and dry AMD ${ }^{(4)}$. Fundus fluorescein angiography (FFA) is an important tool in diagnosing and following the treatment of retinal diseases ${ }^{(5)}$. FFA is not without problems; some patients have contraindications to the dye such as those with hypersensitivity to fluorescein ${ }^{(6)}$. FFA modality is not depth resolved ${ }^{(7)}$. Optical coherence tomography angiography (OCTA) is capable of visualizing the choriocapillaries blood flow, and therefore, may have use in the evaluation of neovascular AMD ${ }^{(8)}$. OCTA is based on repeated consecutive optical coherence tomography (OCT) $\mathrm{B}$-scans at the same section to generate a contrast (decorrelation signal) between static and non-static tissue ${ }^{(9)}$. OCTA does not require administration of intravenous dye such as fluorescein thus avoiding the potential risks which can result in rare adverse events ${ }^{(10)}$.

\section{AIM OF THE WORK}

The aim of this work is to evaluate the role of OCTA in the diagnosis of choroidal neovascularization $(\mathrm{CNV})$ in patients with AMD.

\section{PATIENTS AND METHODS}

In this prospective case series, consecutive subjects were recruited from the retina clinic at Kafr EL Sheikh Ophthalmic center (Kafr El Sheikh, Egypt) from March 2017 to March 2018. The study protocol was adhered to the tenets of the declaration of Helsinki ${ }^{(11)}$ and was approved by the Ethics Board of Al Azhar University. An informed written consent was taken from each participant in the study.

Clinical and instrumental assessments were performed in 32 patients (40 eyes), of whom 20 patients (20 eyes) were diagnosed as wet AMD and 12 patients ( 20 eyes) with dry AMD.

All patients underwent FFA, swept source OCT (SS-OCT) and swept source OCTA (SSOCTA) using Topcon OCT Triton .The three imaging modalities were performed within 7 days of each other. The angioretina of the Topcon OCT Triton utilizes OCTARA algorithm. OCTA acquisition protocol in the macular region consisted of a $6 \times 6 \mathrm{~mm}$ area centered onto the fovea. En face OCTA images were segmented into four layers, namely the 
superficial vascular plexus, deep vascular plexus, outer retina, and choriocapillaries.

The subjects included in this study were patients over 50 years with clinical features of age-related maculopathy, such as soft or hard drusen, pigmentary alterations and macular exudative signs on FFA.

The exclusion criteria included the presence of CNV secondary to retinal diseases other than AMD, myopia greater than 6 Diopters, poor quality images due to media opacity or motion artifact, glaucoma, diabetic retinopathy, history of posterior segment surgery within the last 6 months, history of laser photocoagulation and any contraindication to intravenous fluorescein injection as renal impairment and hypersensitivity.

The following features of neovascular networks were evaluated: location, and extent of visualization. Sensitivity and specificity of the method were assessed separately in a group of 20 CNV eyes and 20 dry AMD eyes. FFA served as the gold standard for CNV diagnosis.

\section{Statistical analysis}

A) Descriptive statistics

1- Quantitative data: mean and standard deviation (SD) were used to measure central tendency and dispersion.

2- Qualitative data: frequency of occurrence was calculated by number and percentage.

B) Analytic statistics:

Comparing both groups was done using:

1- Pearson chi square-test (X2) for qualitative data (12).

2- Student's $T$ test for qualitative data of two independent samples ${ }^{(\mathbf{1 3})}$.

\section{Results}

\section{Clinical results}

FFA diagnosis

Active CNV in group I was classified into occult CNV in 6 eyes (neovascular growth beneath the retinal pigment epithelium (RPE) type 1), classic CNV in 12 eyes (neovascular growth above the RPE - type 2) and mixed CNV in 2 eyes (Table 1).

Table (1): Frequency of types of active CNV (FFA diagnosis)

\begin{tabular}{|l||l|l|}
\hline Type of CNV & Frequency & Percent \\
\hline \hline Occult & 6 & 30 \\
\hline Classic & 12 & 60 \\
\hline Mixed & 2 & 10 \\
\hline Total & 20 & 100 \\
\hline
\end{tabular}

Active $\mathrm{CNV}$ in group I was classified according to its site into subfoveal in 15 eyes, juxtafoveal in 2 eyes and extrafoveal in 3 eyes (Table 2).

Table (2): Site of CNV (FFA diagnosis)

\begin{tabular}{|c||c|c|}
\hline Variable & Frequency & Percent \\
\hline \hline Subfoveal & 15 & 75 \\
\cline { 2 - 3 } Juxtafoveal & 2 & 10 \\
\cline { 2 - 3 } Extrafoveal & 3 & 15 \\
\hline \hline Total & 20 & 100 \\
\hline
\end{tabular}

\section{OCTA diagnosis}

Occult CNV lesions (type I on OCT) were best visualized on the SS-OCTA en face projection of the choriocapillaris (Figure 1), whereas classic CNV lesions (type II on OCT) were best visualized on the SS-OCTA en face projection of the outer retina (Figure 2). OCTA enabled accurate localization of neovascular networks with respect to the RPE layer. Motion artifact was detected in $18 \%$ of patients.

Features detected on SS-OCTA images of active $\mathrm{CNV}$ lesion were vascular anastomosis and looping within the $\mathrm{CNV}$, increased density of tiny capillaries, extensive arborization, , and a hollow zone around the lesion separating it from the surrounding normal vasculature. All CNV lesions in group I showed signs of activity on FFA and on SS-OCTA (except one case which was false negative on OCTA).
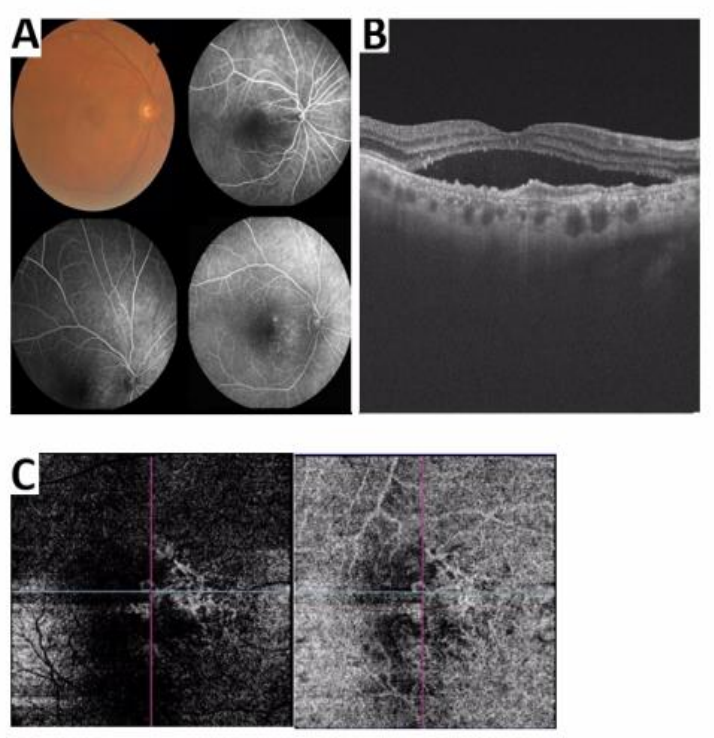

Figure 1:

A) Color photo and FFA of the right eye of a 65-year-old male with exudative AMD and occult CNV.

B) SS-OCT shows that RPE in the sub-foveal area is irregular, thickened, and raised up by a moderately hyper-reflective lesion associated 
withaccumulationof sub retinal fluid, which indicates type I CNV.

C) En face SS-OCTA image in a $6 \times 6 \mathrm{~mm}$ field. Left: outer retina. Right: choriocapillaris.

The lesion demonstrates homogenous network of tiny interlacing capillaries with occasional larger vessels displayed as jet-white streaks. The entire complex is surrounded by a dark halo. The extent of arborization of the neovascular complex is most revealed at the choriocapillaris projection compared to the outer retina projection.
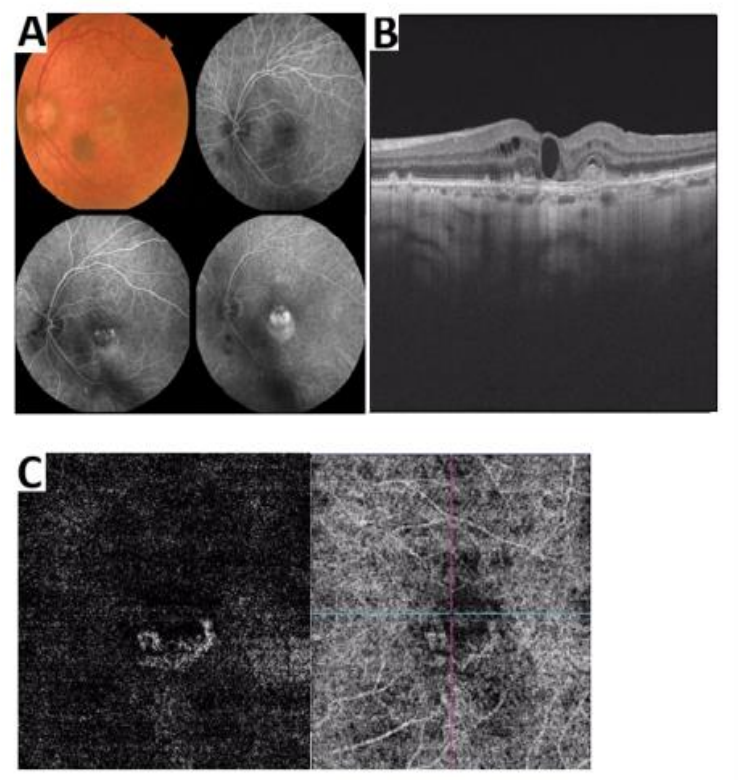

Figure 2:

A) Color photo and FFA of the left eye of a 70year-old male with exudative AMD and classic $\mathrm{CNV}$.

B) SS-OCT shows a hyper-reflective nodular lesion located entirely above the RPE. The lesion resulted in disruption of the retinal layers and is associated with gross intra-retinal edema which indicates type II active CNV.

C) En face SS-OCTA image in a $6 \times 6 \mathrm{~mm}$ field. Left: outer retina. Right: choriocapillaris. The extent of arborization of the neovascular complex is most revealed at the outer retina projection compared to choriocapillaris the projection (type II active CNV).

Having analyzed the results, we found that OCTA was positive in 19 eyes with confirmed CNV, which means that the method's sensitivity is $95 \%$ (Table 3). A false negative case with no decorrelation signal on OCTA was due to subretinal hemorrhage (Figure 3). In group II of 20 eyes OCT angiography showed no decorrelation signal at outer retina or choriocapillaris which means that the method's specificity was $100 \%$ (Figure 4).
Table (3): Frequency of active CNV secondary to AMD by OCTA diagnosis.

\begin{tabular}{|c|c|c|}
\hline OCTA & Frequency & Percent \\
\hline \hline Active CNV & 19 & 47.5 \\
\hline No CNV & 21 & 52.5 \\
\hline Total & 40 & 100 \\
\hline
\end{tabular}

Structural OCT was positive in 20 eyes with confirmed CNV, which means that the method's sensitivity is $100 \%$. In group II Structural OCT showed no neovascularization which means that the method's specificity was $100 \%$ (Table 4).

Table (4): Frequency of active CNV secondary to AMD by OCT diagnosis.

\begin{tabular}{|c|c|c|}
\hline OCT & Frequency & Percent \\
\hline \hline Active CNV & 20 & 50 \\
\hline No CNV & 20 & 50 \\
\hline \hline Total & 40 & 100 \\
\hline
\end{tabular}
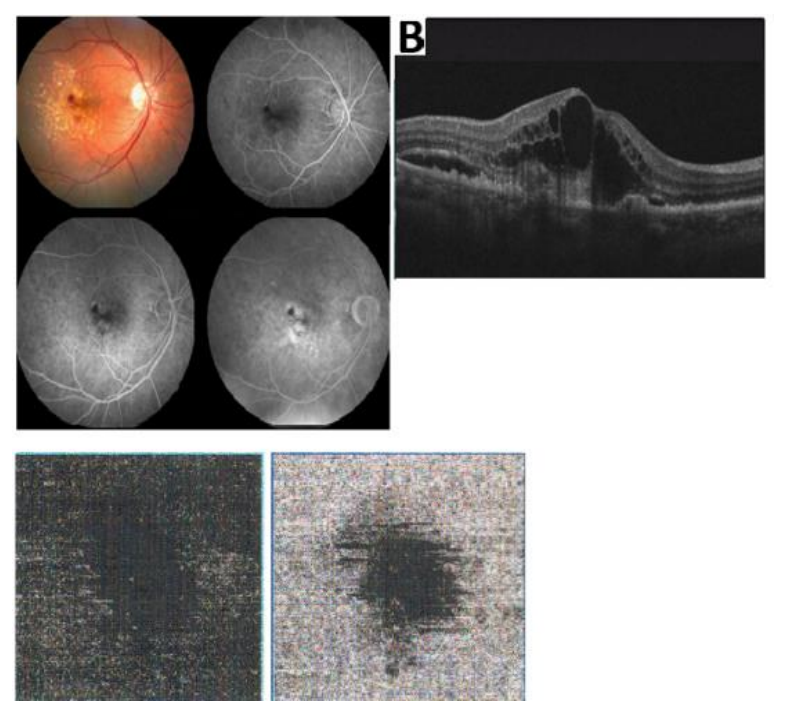

Figure 3:

A) Color photo and FFA of the right eye of a 79year-old female with exudative AMD and minimally classic $\mathrm{CNV}$.

B) OCT shows sub and intra-retinal fluid and subretinal hemorrhage.

C) En face SS-OCTA image in a 6x6 mm field. Left: outer retina. Right: choriocapillaris

There is no dcorrelation signal (False negative). 


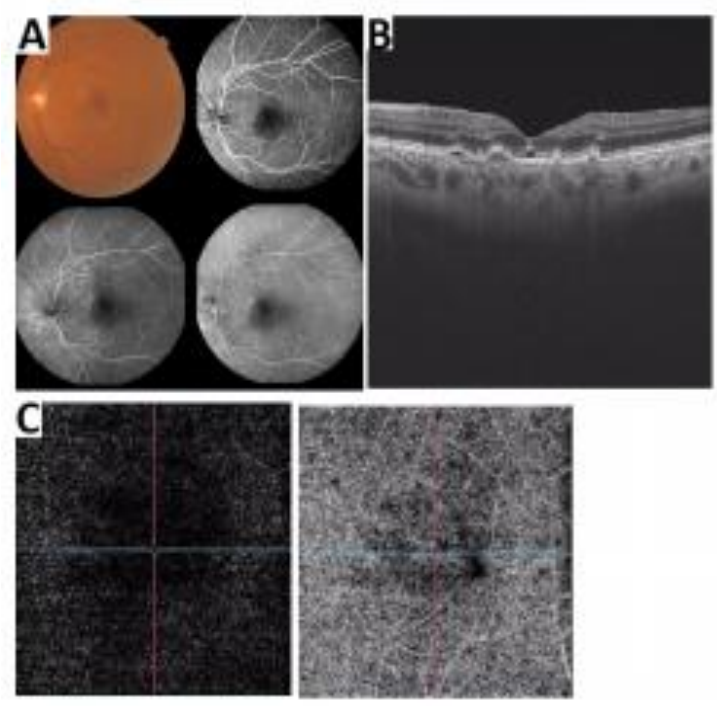

Figure 4:

A) Color photo and FFA of the left eye of a 65year-old male with dry AMD.

B) SS-OCT image in radial scan showing soft drusen.

C) En face SS-OCTA image of the same eye taken in a $6 \times 6 \mathrm{~mm}$ field. No decorrelation signal at the outer retina or the choriocapillatis.

\section{DISCUSSION}

The management of $\mathrm{CNV}$ requires using FFA combined with OCT to obtain functional and anatomical information on the lesion that help to establish the diagnosis, to predict the visual outcome of therapy, and for follow-up. FFA reflects the various pattern of activity of CNV (dye leakage) ${ }^{(14)}$.

OCT is a noninvasive, depth-resolved imaging technique. Although OCT aids the clinician in visualizing the anatomic changes that impact vision, it offers poor contrast between small blood vessels and static tissue in most retinal layers. As a result, structural OCT is not used clinically to identify vascular changes such as capillary dropout or pathologic new vessel growth in $\mathrm{AMD}^{(15)}$.

OCTA is a novel and noninvasive imaging tool, which allows visualization of retinal microvasculature by detecting intravascular blood flow without any dye injection. This new technique may be used in daily clinical ophthalmology practice and may, in the future, replace invasive techniques ${ }^{(\mathbf{1 6})}$.

Current study aimed to assess the ability of the OCTA technique in detecting active CNV compared to FFA as a gold standard and to determine efficacy (sensitivity and specificity) of SS-OCTA compared to FFA.

In our study OCTA has proved reliable in distinguishing between the two types of CNV: occult and classic, new blood vessels growing beneath or above the RPE, respectively.

In our study, we detected a dark halo surrounding active $\mathrm{CNV}$ lesions and that was displayed as a hypointense clear zone on SSOCTA images. This finding is consistent with Jia et al..$^{(9)}$, Kuehlewein et al. ${ }^{(17)}$, and Coscas et $a l .{ }^{(18)}$ who reported the same sign.

An explanation for this dark halo is that $\mathrm{CNV}$ tends to develop a region of choriocapillaris alteration caused by impaired flow to compensate for ischemia. The region of choriocapillaris alteration is located underneath the CNV and extends beyond its margins in the form of a ring or halo and appears as hypointense or silent area on SS-OCTA images due to reduced blood flow ${ }^{(\mathbf{1 4})}$.

In our study the sensitivity and specificity of OCTA in detecting the CNV secondary to nAMD was $95 \%$ and $100 \%$ respectively, higher than that of Faridi A et al ${ }^{(19)}$. This higher result of our study was due to low number of cases with subretinal hemorrhage, only one false negative eye with no decorrelation signal on en face OCTA due to subretinal hemorrhage in our study. This finding is consistent with other studies, which have reported a decreased ability of OCTA to detect CNV in eyes with subretinal hemorrhage. Moult et al $^{\left({ }^{(20)}\right.}$ reported in their series that the single case in which OCTA revealed false-negative result had dense subretinal hemorrhage that caused severe attenuation of the SS-OCT signal. Faridi et al. ${ }^{(19)}$ concluded that sensitivity of en face OCTA improved to $94 \%$ if eyes with subretinal hemorrhage were excluded. Jia et $\boldsymbol{a l} .{ }^{(9)}$ demonstrated the ability of OCTA to detect and quantify $\mathrm{CNV}$ in 10 patients with wet $\mathrm{AMD}$ where OCTA provided better visualization of the neovascular network with respect to FA, as images were not obscured by subretinal hemorrhage or other artifacts. De Carlo et al. ${ }^{(7)}$ reported that the sensitivity and specificity of OCTA in detecting CNV secondary to wet AMD were (4/8) 50\% and (20/22) 91\%, respectively. Low sensitivity was due to small sample size and blockage from large amounts of retinal hemorrhage in some patients. Nikolopoulou et $\boldsymbol{a l} .{ }^{(21)}$ reported that the sensitivity and specificity of OCTA in detecting CNV secondary to wet AMD $88 \%$ \& $90 \%$ respectively.

In our study OCTA showed high sentivity in detecting type I CNV. This result was partially in discordance with Nikolopoulou et al. ${ }^{(21)}$ who stated that OCTA would display worse sensitivity in naïve $\mathrm{CNV}$, due to undetectable 
flow inside the small peripheral branches of the neovascular complex.

In our study active CNV on OCTA showed well-defined complexes, dark halo around the lesion and numerous tiny anastomotic capillaries with thin walls and small diameter. There was excellent level of correlation in treatment decision based on OCTA compared to FFA. This result is consistent with findigs of Coscas et al. ${ }^{(18)}$ and Spaide et al..$^{(22)}$. Coscas et al. ${ }^{(18)}$ had compared the OCTA with traditional multimodal imaging in patients with wet AMD and found that there was high interobserver agreement both for treatment decision in conventional multimodal and for pattern I (active CV) or pattern II (inactive CNV) definition in OCTA imaging analysis.

SS-OCT can provide clues suggesting the presence of $\mathrm{CNV}$, such as the presence of SRF, IRF, and subretinal hyperreflective material, and allows identification of areas where the RPE is separated from BM.

In our study the sensitivity and specificity of OCT alone compared to FFA was $100 \%$ nearly similar to Faridi et al. ${ }^{(\mathbf{1 9 )}}$ who reported a sensitivity $100 \%$ and specificity $97.5 \%$. This high sensitivity may be attributed to the use of SS-OCT which gives more image resolution so pathological features are not easily overlooked compared to TD-OCT and SD-OCT.

Other studies which compared OCT to FFA reported a wide range of sensitivity and specificity respectively: $90 \%$ and $47 \%{ }^{(23)}, 94 \%$ and $98 \%{ }^{(24)}$ and, $100 \%$ and $80 \%{ }^{(25)}$.

In our study, the sensitivity of OCT along with en face OCTA in detecting the active CNV secondary to AMD compared to FFA was $100 \%$. This study agreed with study of Faridi et al..$^{(19)}$ who showed the same results.

\section{CONCLUSION}

OCTA is reliable in distinguishing between occult and classic CNVs in neovascular AMD patients. OCTA allowed the diagnosis of CNVs in most of the cases analyzed, with exception of cases presenting subretinal hemorrhages. With regard to $\mathrm{CNV}$, occult or classic, OCT angiography has been shown to have $95 \%$ sensitivity and $100 \%$ specificity.

Combined SS-OCT and SS-OCTA are capable of providing accurate anatomical information on the location of the CNV.

\section{REFERENCES}

1. Bird A, Frederick L, Wilkinson CP et al. (2013): Clinical classification of age- related macular degeneration. Ophthalmology, 120(4): 844-51.

2. Reynolds R, Seddon JM, Yu Y et al. (2011): Risk models for progression to advanced age-related macular degeneration using demographic, environmental, genetic, and ocular factors. Ophthalmology, 118:2203-11.

3.Mariotti SP and Pascolini D (2012): Global estimates of visual impairment. $\mathrm{Br} \mathrm{J}$ Ophthalmol., 96(5): 614-8.

4. Cook HL, Patel PG and Tufail A (2008): Age-related macular degeneration: diagnosis and treatment. British Medical Bulletin, 85: 127-49.

5. Jennifer I (2013): Age-Related Macular Degeneration. $3^{\text {rd }}$ ed, CRC Press.

6. Xu K, Tzankova V, Li C et al. (2016): Intravenous fluorescein angiography associated adverse reactions. Can J Ophthalmol., 51(5): 321-5.

7. De Carlo TE, Bonini MA, Chin AT et al. (2015 Spectral-domain optical coherence tomography angiography of choroidal neovascularization.

Ophthalmology,2015;122(6): 1228-38.

8. Bruno L(2015): Clinical OCT Angiography Atlas. $1^{\text {st }}$ ed, Jaypee Brothers Medical Publishers.

9. Jia Y, Bailey ST, Wilson DJet al. (2014): Quantitative optical coherence tomography angiography of choroidal neovascularization in age-related macular degeneration. Ophthalmology, 121(7):1435-44.

10. Su Z, Ye P, Teng Y et al. (2012): Adverse reaction in patients with drug allergy history after simultaneous intravenous fundus fluorescein angiography and indocyanine green angiography. Ocul Pharmacol Ther., 28(4): 410-3.

11. World Medical Association Declaration of Helsinki (2013): Ethical Principles for Medical Research Involving Human Subjects. JAMA., 310(20):2191-2194.

12. Richard Mankiewicz (2004): The story of Mathematics (Paperback ed.). Princeton University Press.

13. NIST (2006): Engineering Statistics Handbook-Chi-Squared Distribution. https://www.itl.nist.gov/div898/handbook/ed a/section3/eda3666.htm

14. Moussa M, Mahmoud $\mathbf{L}$ and Khalid $\mathbf{H}$ (2017):Imaging choroidal neovascular membrane using en face swept-source optical coherence tomography 
angiography. Dove Medical Press Journal, 11:1859-69.

15. Simon S, Yali J, Miao Zet al. (2016): Optical Coherence Tomography Angiography. Investigative Ophthalmology \& Visual Science, 57:27-36.

16. Souedan V, Souied E, Caillaux $\mathrm{V}$ et al. (2018): Sensitivity and specificity of optical coherence tomography for detection of choroidal neovascularization in real-life practice and varying retinal expertise level. IntOphthalmol., 38:1051-60.

17. Kuehlewein L, Bansal M, Lenis TL et al. (2015): Optical coherence tomography angiography of type 1 neovascularization in age-related macular degeneration. Am J Ophthalmol., 160:739-48.

18. Coscas GJ, Lupidi M, Cagini C et al. (2015): Optical Coherence Tomography Angiography versus traditional multimodal imaging in assessing the activity of exudative age-related macular degeneration. A new diagnostic challenge. Wolters Kluwer:Retina, 35:2219-28.

19. Faridi A, Jia Y, Gao S et al. (2017): Sensitivity and Specificity of OCT Angiography to Detect Choroidal Neovascularization. AAO., 1:294-303.

20. Moult E, Choi W, Waheed NK et al. (2014), Ultrahigh-speed swept-source OCT angiography in exudative AMD. Ophthalmic Surg Lasers Imaging Retina, 45(6):496-505.
21.Nikolopoulou E ,LorussoM, Ferrari LM et al. (2018): Optical Coherence Tomography Angiography versus Dye Angiography in Age-Related Macular Degeneration: Sensitivity and Specificity Analysis. BioMed Research International,672:1-7.

22. Spaide RF, Klancnik JM, Cooney J (2015): Retinalvascular layers imaged by fluorescein angiography and optical coherence tomography angiography. JAMA Ophthalmol., 133(1): 45-50.

23.Khurana RN, Dupas B and Bressler NM (2010): Agreement of time-domain and spectral-domain optical coherence tomography with fluorescein leakage from choroidal neovascularization.Ophthalmology,117(7): 1376-1380.

24.Nils F Mokwa, Tina Ristau, Pearse A Keane et al. (2013): Clinical Study Grading of Age-Related Macular Degeneration: Comparison between Color Fundus Photography, Fluorescein Angiography, and Spectral Domain Optical Coherence Tomography. Hindawi Journal of Ophthalmology,385:1-6.

25.Wilde C, Patel M, Lakshmanan A et al. (2015): The diagnostic accuracy of spectraldomain optical coherence tomography for neovascular inage-related macular degeneration: a comparison with fundus fluorescein angiography. Eye, 29:602-10 